\section{Using partitioned heritability methods to explore genetic architecture}

\author{
Luke M. Evans and Matthew C. Keller
}

In their recent Review (Genetic architecture: the shape of the genetic contribution to human traits and disease. Nat. Rev. Genet. 19, 110-124 (2018) $)^{1}$, Timpson et al. discuss the importance of genetic architecture in human disease and some methods to measure and understand genetic architecture. Their discussion of genetic architecture assessment focuses primarily on genome-wide association studies (GWAS), whole-exome sequencing and whole-genome sequencing approaches to identify variants statistically linked to phenotypes of interest. In addition to their excellent treatment of these tools, they mention only briefly recent approaches that use genomewide markers to explore the heritability of traits, including partitioned heritability, citing a relevant 2013 review of heritability methods $^{2}$. However, rapid advances since 2013 have made these approaches particularly relevant to assessing genetic architecture. Here, we highlight three areas where genome-wide methods can add to the understanding of genetic architecture.

First, partitioned variance component models using restricted maximum likelihood (REML) can explore the relative contribution of variants across the allele frequency spectrum or relevant annotations to phenotypic variance. These methods (termed genomic relationship matrix-REML, or GREML ${ }^{3}$ ) estimate the genetic variance attributable to single nucleotide polymorphisms (SNPs) partitioned into various bins or annotations, thereby estimating the contribution of particular gene networks or annotations ${ }^{4}$ or, in combination with deep imputation panels and large sample sizes, reliably partitioning phenotypic variance due to common, low-frequency or very rare genetic variants 5 . Specifically, partitioning by linkage disequilibrium (LD) and minor allele frequency (LDMS) can accurately estimate the contribution of variants across the frequency spectrum ${ }^{6}$. These approaches have now also been extended to infer the role of purifying selection across the genome $e^{7,8}$ and gene-environment (GxE) interactions ${ }^{9}$. Notably, they can estimate genetic variance even when no or few genome-wide significant GWAS hits have been identified (for an example see REF. ${ }^{10}$ ).

Second, computationally efficient, partitioned LD score regression (LDSC) ${ }^{11-13}$ relates the LD tagging of common SNPs to their GWAS summary statistics to estimate heritability. By quantifying the heritability enrichment of particular annotations (and not limiting to coding variation), partitioned LDSC can elucidate functional domains that contribute to phenotypic variation, illuminating differences in the genetic architecture of traits, such as levels of triglycerides and lowdensity lipoprotein (LDL) ${ }^{13}$, and evolutionary connections, such as purifying selection and allele age ${ }^{11}$.

Last, GREML and LDSC can be used to explore the genetic correlation of traits ${ }^{14,15}$. This may be due to pleiotropy of variants, to close proximity and linkage of multiple variants that have an impact on different traits, or to long-range gametic phase disequilibrium caused by cross-trait assortative mating. Bivariate analyses have been used to clarify expected genetic correlations between traits, for instance, hypertension and type 2 diabetes mellitus ${ }^{14}$ or high-density lipoprotein (HDL) and triglyceride levels ${ }^{15}$. However, these approaches have also highlighted genetic correlations that may have been previously unexpected, for instance, substantial positive genetic correlations among bipolar disorder, major depression and schizophrenia ${ }^{10,15}$.

Marker-based heritability estimation methods, used to partition genetic variance among annotations or allele frequencies, or to estimate genetic correlations, are a powerful and rapidly advancing set of tools that can aid our understanding of genetic architecture, help guide study design and support the exploration of the forces that shape genetic variation.
Luke M. Evans ${ }^{1 *}$ and Matthew C. Keller ${ }^{1,2}$

${ }^{1}$ Institute for Behavioral Genetics, University of Colorado, Boulder, CO, USA.

${ }^{2}$ Department of Psychology and Neuroscience, University of Colorado, Boulder, CO, USA.

*e-mail: luke.m.evans@colorado.edu doi: $10.1038 / \mathrm{nrg} .2018 .6$ Published online 14 Feb 2018

1. Timpson, N. J. et al. Genetic architecture: the shape of the genetic contribution to human traits and disease. Nat. Rev. Genet. 19, 110-124 (2018).

2. Tenesa, A. \& Haley, C. S. The heritability of human disease: estimation, uses and abuses. Nat. Rev. Genet. 14, 139-149 (2013).

3. Yang, J. et al. Common SNPs explain a large proportion of the heritability for human height. Nat. Genet. 42, 565-569 (2010)

4. Lee, S. H. et al. Estimating the proportion of variation in susceptibility to schizophrenia captured by common SNPs. Nat. Genet. 44, 247-250 (2012).

5. Yang, J. et al. Genetic variance estimation with imputed variants finds negligible missing heritability for human height and body mass index. Nat. Genet. 47, 1114-1120 (2015).

6. Evans, L. M. et al. Comparison of methods that use whole genome data to estimate the heritability and genetic architecture of complex traits. Preprint at bioRxiv https://doi.org/10.1101/115527 (2017). 7. Speed, D. et al. Re-evaluation of SNP heritability in complex human traits. Nat. Genet. 49, 986-992 (2017).

8. Zeng, J. et al. Widespread signatures of negative selection in the genetic architecture of human complex traits. Preprint at bioRxiv https://doi. org/10.1101/145755 (2017).

9. Robinson, M. R. et al. Genotype-covariate interaction effects and the heritability of adult body mass index. Nat. Genet. 49, 1174-1181 (2017).

10. Lee, S. H. et al. Genetic relationship between five psychiatric disorders estimated from genome-wide SNPs. Nat. Genet. 45, 984-994 (2013).

11. Gazal, S. et al. Linkage disequilibrium dependent architecture of human complex traits reveals action of negative selection. Nat. Genet. 49, 1421-1427 (2016)

12. Bulik-Sullivan, B. K. et al. LD score regression distinguishes confounding from polygenicity in genome-wide association studies. Nat. Genet. 47 291-295 (2015).

13. Finucane, H. K. et al. Partitioning heritability by functional annotation using genome-wide association summary statistics. Nat. Genet. 47, 1228-1235 (2015).

14. Lee, S. H. et al. Estimation of pleiotropy between complex diseases using single-nucleotide polymorphism-derived genomic relationships and restricted maximum likelihood. Bioinformatics 28, 2540-2542 (2012).

15. Bulik-Sullivan, B. et al. An atlas of genetic correlations across human diseases and traits. Nat. Genet. 47, 1236-1241 (2015).

Competing interests

The authors declare no competing interests. 